\title{
Fatigue Lives of Power Plant Structures Due to Load Sequence Effects Originating from Fluctuating Production of Renewable Energy
}

\author{
Michael Vormwald ${ }^{1, *}$, Alexander Bosch ${ }^{1}$, Sophie Schackert ${ }^{2}$, Christoph Schweizer ${ }^{2}$ \\ ${ }^{1}$ Technische Universität Darmstadt, Materials Mechanics Group, 64287 Darmstadt, Germany \\ ${ }^{2}$ Fraunhofer Institute for Mechanics of Materials IWM, 79108 Freiburg, Germany
}

\begin{abstract}
The change in operation of power plants due to the increasing use of renewable energies causes additional stresses to the components by a high number of smaller load cycles. This fact results in a demand for validated new approaches to estimate fatigue life especially for welded joints that are the weak parts within the piping. The components are operated in the LCF regime where short fatigue crack growth determines the life. Therefore, a non-linear fracture mechanics based approach is appropriate. For the development and validation of the model, an experimental campaign was performed including fatigue tests of smooth specimens with various microstructures of $\mathrm{X} 6 \mathrm{CrNiNb} 18-10$ (AISI 347) as well as specimens containing mechanical and microstructural notches. Experiments are performed with all types of specimens with an increasing complexity from constant to variable amplitude loading, the latter also applied as pseudorandom sequence derived from measurements in power plants. The developed non-linear fracture mechanics based model uses the effective cyclic J-Integral normalized to the crack length to replace crack growth calculation by a linear damage accumulation. To consider the loading history an algorithm for the calculation of crack opening and crack closure is used. The advantages of this approach are evident when comparing calculated with experimentally determined fatigue lives. Remaining differences serve for further considerations of how to improve the life prediction for variable amplitudes.
\end{abstract}

\section{Introduction}

The fluctuating production of renewable energies compared with a cyclically varying energy consumption mainly induced by day-night-rhythm led to a flexible operation of conventional power plants. Conventional power plants have to cover the gaps in energy consumption between day-night-rhythm, but even between a windy cloudless summer day when solar and wind are able to cover the whole energy needs and a windless cloudy winter day when solar and wind do not contribute the production of energy. Without the possibility to store the surplus of energy for peak-periods, conventional power plants still need to be present. The

* Corresponding author: vormwald@wm.tu-darmstadt.de 
reasons for this dilemma can be discussed controversially, but the consequences need to be quantified on basis of scientific findings. Life assessments can be based on various approaches and a variety of influence factors might necessarily be taken into account. Depending on the effort, the user respectively the operator of a power plant can gain a more realistic assessment of a component. For the assessment of life under variable amplitude loading a selection of methods is presented and discussed in this paper. For validation, a large number of tests under variable amplitude loading were performed with various specimens.

\section{Material, Specimens and Testing}

The material in the focus of investigations is the austenitic stainless steel 1.4550 (X6CrNiNb18-10) which is commonly used for the piping of conventional power plants. Four different types of specimens are tested: unnotched specimens made of base and heataffected-zone material, the latter produced by a Gleeble welding simulator (see [15] for details of the machine), notched specimens of base material and welded specimens. Base material and Gleeble specimens are tested at different temperatures between ambient temperature and $350^{\circ} \mathrm{C}$. Welded and notched specimens are tested at $180^{\circ} \mathrm{C}$ which is the average of the temperatures measured during shutdown of a power plant at the component of interest (see Fig. 1). Three test types are performed: fatigue tests under constant amplitude loading, under two stage amplitude loading and under eight stage amplitude loading. The eight stage amplitude loading has to be distinguished into block tests, operational load tests and operational load tests with a superimposed thermal cycle (operational load TMF). Two stage amplitude loading tests were performed by the sequence of one big cycle followed by a 10,100, 1000 or 10000 cycles of a much lower level. The tests under eight stage amplitude loading are performed with the amplitudes and their frequency of occurrence derived from the temperature measurement of a component during shutdown of a nuclear power plant (see Fig. 1, left). The measured temperatures were processed to a local stress and strain history [1]. Based on this data, the authors counted the cycles with a rainflow algorithm [2] and created a load spectrum by the Sum of two Gaussian like distributed (SoftGild) load spectra according to measured load spectrum (see Fig. 1, right).
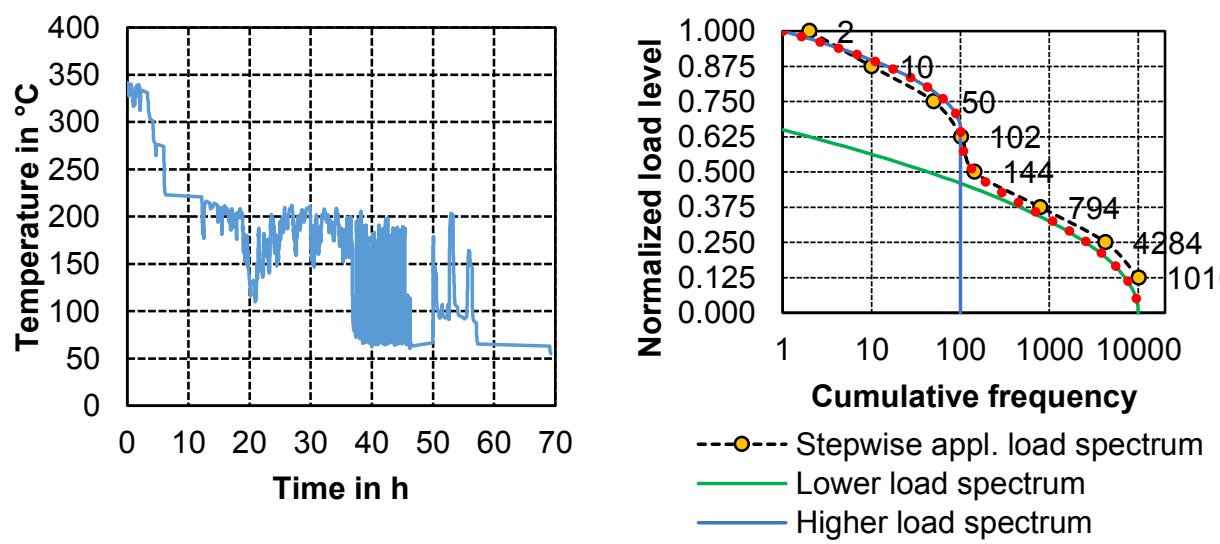

Fig. 1. Measured temperature for shutdown period of 3 days [1] (left); Investigated SOFTGILD (sum of two Gaussian like distributed load spectra) load spectrum by sum of higher and lower load spectrum, derived load spectrum for stepwise application by 8 steps (right)

The higher portion of SoftGild load spectrum contains about $10^{2}$ cycles, the lower portion about $10^{4}$ cycles). For the block tests, the amplitudes appear in blocks of ascending and 
descending sequence, for the operational load tests the amplitudes appear in a pseudo-random sequence. The latter sequence is superimposed with a thermal cycle of $50-350^{\circ} \mathrm{C}$ for the operational load TMF tests. The highest amplitude of variable amplitude loading tests was always performed under a controlled strain ratio of $\mathrm{R}=-1$. The position of the smaller amplitudes within the largest cycle was varied. The three investigated variations consist of (a) mean strain equal to zero, (b) highest possible mean strains resulting in a sequence where each peak has the maximum strain $\left(\varepsilon_{\max }=\right.$ const. $)$, and $(\mathrm{c})$ lowest possible mean strains resulting in a sequence where each valley has the minimum strain $\left(\varepsilon_{\min }=\right.$ const. $)$.

\section{Damage Calculation Methods}

Damage calculations for the two stage test have been performed applying the linear damage accumulation rule (Palmgren-Miner's hypothesis $[3,4]$ ) in connection with several damage parameters. First, the life is calculated by considering only the strain ranges. Second, life is calculated using the damage parameter $P_{\mathrm{SWT}}$, additionally taking mean stresses into account. Finally, the damage parameter $P_{\mathrm{J}}$ containing the cyclic effective J-Integral has been applied. The linear damage accumulation rule assumes, that the ratio of the applied number of cycles, $n_{i}$, of each load level $i$ (in terms of strain ranges or a damage parameter) to the number of load cycles to failure $N_{i}$ under constant amplitude loading with load level $i$ is summed,

$$
D=\sum_{i=1}^{k} \frac{n_{i}}{N_{i}} .
$$

Failure is assumed for $D=1$. Hence, a load spectrum with a number of cycles $H_{0}=\sum n_{i}$ and a damage sum of $D_{\mathrm{H}_{0}}$ can be applied $H$ times until failure occurs theoretically:

$$
H=\frac{1.0}{D_{\mathrm{H}_{0}}}
$$

Furthermore, the experimentally investigated endurance limit under constant amplitude loading of $\varepsilon_{\mathrm{e}}=0.1937 \%$ is considered for each damage calculation.

\subsection{Damage Calculations Based on Strain Amplitudes}

For the damage calculation based on strain ranges, performed with the linear damage accumulation rule, the derived strain life curve in terms of the Manson-Coffin-Basquin [5-7] equation is used as reference to calculate the number of cycles to failure for each load level.

\subsection{Damage Calculations Based on Strain Amplitudes and Mean Stresses}

In addition to previous damage calculation method with the pure strain ranges, the commonly used damage parameter $P_{\text {SWT }}$ [8] is introduced by Equation 3. This damage parameter takes the mean stresses of each load cycle into account, load cycles with identical strain ranges and different mean stresses lead to different amounts of damage.

$$
P_{\mathrm{SWT}}=\sqrt{\left(\sigma_{\mathrm{a}}+\sigma_{\mathrm{m}}\right) \varepsilon_{\mathrm{a}} E}
$$

The damage parameter $P_{\mathrm{SWT}}$ uses the Manson-Coffin-Basquin equation for the mathematical description of the damage parameter life curve:

$$
P_{\mathrm{SWT}}=\sqrt{\sigma_{f}^{\prime 2}(2 N)^{2 b}+\sigma_{f}^{\prime} \varepsilon_{f}^{\prime} E(2 N)^{b+c}}
$$

\subsection{Damage Calculations Based on Fracture Mechanics Short Crack Growth}


In contrast to empirical approaches, a mechanism-oriented concept for describing thermomechanical fatigue should be based on fracture mechanics for short cracks. The effective cyclic J-Integral $\Delta J_{\text {eff }}$ is used as a measure for the fatigue crack growth rate based on a Paris type law [9] with the coefficient $C$ and the exponent $m$ :

$$
\frac{\mathrm{d} a}{\mathrm{~d} n}=C \cdot \Delta J_{\mathrm{eff}}^{m}
$$

The $P_{\mathrm{J}}$-concept uses an approximation formula for the effective cyclic J-Integral for halfpenny shaped surface cracks [10]. The term "effective" means that crack closure is taken into account. Effective stress and strain ranges are calculated by the difference between the value of the upper reversal point to the value when the crack closes, Fig. 2 (left). The effective cyclic J-Integral can be written as a function of these effective ranges, material parameters and the crack length $a$,

with

$$
\Delta J_{\mathrm{eff}}=\left[1.24 \frac{\Delta \sigma_{\mathrm{eff}}^{2}}{E}+\frac{1.02}{\sqrt{n^{\prime}}} \Delta \sigma_{\mathrm{eff}}\left(\Delta \varepsilon_{\mathrm{eff}}-\frac{\Delta \sigma_{\mathrm{eff}}}{E}\right)\right] a,
$$

$$
\Delta \sigma_{\mathrm{eff}}=\left(\sigma_{\max }-\sigma_{\mathrm{cl}}\right) ; \Delta \varepsilon_{\mathrm{eff}}=\left(\varepsilon_{\max }-\varepsilon_{\mathrm{cl}}\right)
$$

In order to avoid time consuming crack growth calculations, the damage parameter, $P_{\mathrm{J}}$, is introduced,

$$
P_{\mathrm{J}}=\frac{\Delta J_{\mathrm{eff}}}{a}
$$

For constant amplitude loadings, the fatigue life curve can be expressed for values of $P_{\mathrm{J}}$ above the endurance limit $P_{\mathrm{J}, \mathrm{e}}$ by the following power-law function:

$$
N\left(P_{\mathrm{J}}\right)=Q P_{\mathrm{J}}^{-m} \text { for } P_{\mathrm{J}}>P_{\mathrm{J}, \mathrm{e}}
$$

The exponent $m$ is equal to the exponent of the Paris law. The state of failure denoted by $N$ is connected with a technical crack length $a_{\text {end }}$. By using $a_{\text {end }}$ and the parameters $C, m$ and $Q$, the initial crack length $a_{0}$ can be recalculated by a backward integration of the crack growth law:

$$
a_{0}=\left(a_{\text {end }}^{1-m}-(1-m) C \cdot Q\right)^{\left(\frac{1}{1-m}\right)}
$$

In this context, the initial crack length is a fictitious crack length and does not have to coincide with the "real" initial crack length. The effective stress and strain ranges as well as the point of crack closure are calculated based on Newman's crack closure model [12]. The crack opening stress $\sigma_{\text {op }}$ depends on the maximum stress $\sigma_{\max }$ at the upper point of reversal, the stress-ratio $R$ and an average yield stress $\sigma_{0}$ which is defined by the mean value of cyclic yield strength and ultimate tensile strength:

$$
\sigma_{0}=0.5\left(R_{\mathrm{p} 0.2}^{\prime}+R_{\mathrm{m}}\right) \text {. }
$$

Based on experimental observations the independency of crack opening from crack length is confirmed [13]. Furthermore, assuming that crack opening and crack closing remote strains are approximately equal, with the calculated crack opening stress $\sigma_{\mathrm{op}}$ (see Equation 16) and the Ramberg-Osgood equation it is possible to calculate the crack closure/opening strain with Equation 17 as well as the crack closure stress with Equation 18. With these values the $P_{\mathrm{J}}$ damage life curve can be created by determining the parameters $Q$ and $m$ from Equation 8 . For a damage calculation, the coefficient $\mathrm{C}$ and a threshold value for effective cyclic $\mathrm{J}$ Integral $\Delta J_{\text {eff,th }}$ are needed. These two parameters can be approximated [14] by:

$$
\begin{gathered}
C=10^{-5}\left(5 \cdot 10^{5} \frac{1}{\mathrm{~mm}}\right)^{m} E^{-m}, \\
\Delta J_{\mathrm{eff}, \mathrm{th}}=\frac{E}{5 \cdot 10^{6}} \mathrm{~mm} .
\end{gathered}
$$


Because of the fact, that the initial crack length $a_{0}$ is a fictitious crack length, the initial endurance limit for the damage parameter $P_{\mathrm{J}, \mathrm{e}, 0}$, does not correspond to the threshold value for the cyclic J-Integral $\Delta J_{\text {eff,th }}$ (determined for long cracks) by:

$$
P_{\mathrm{J}, \mathrm{e}, 0} \cdot a_{0} \neq \Delta \mathrm{J}_{\mathrm{eff}, \mathrm{th}}
$$

To re-establish equality in Equation 13, a microstructural constant for short crack threshold description is introduced:

$$
l^{*}=\frac{\Delta J_{\mathrm{eff}, \mathrm{th}}}{P_{\mathrm{J}, \mathrm{e}, 0}}-a_{0 .}
$$

With proceeding crack growth, i.e. increasing crack length, the microstructural constant can be neglected in contrast to the crack length and it can be written:

$$
\text { For } l^{*} \ll a: P_{\mathrm{J}, \mathrm{e}} \cdot a \approx P_{\mathrm{J}, \mathrm{e}} \cdot\left(a+l^{*}\right)=\Delta J_{\mathrm{eff}, \mathrm{th}}
$$

The $P_{\mathrm{J}}$ Wöhler curve describes well the experimental results under constant amplitude loading of the unnotched specimens, Fig. 2 (right).
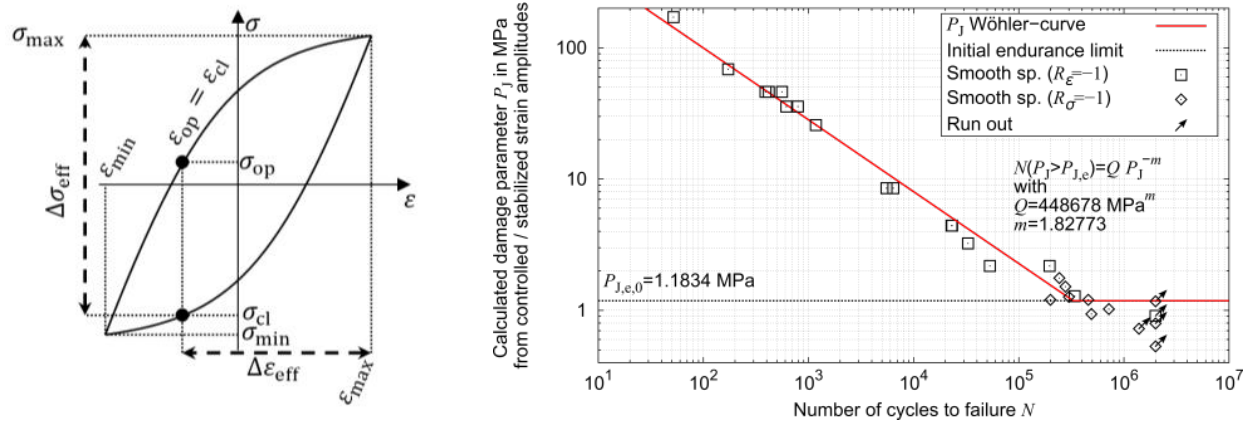

Fig. 2. Definition of effective stress and strain-ranges (left); PJ-Wöhler-curve fitted to experimental results of unnotched specimens under constant amplitude loading (right)

For a damage calculation for variable amplitude loading, the following steps are necessary for each closed cycle [13]:

1. Calculation of stress at crack opening by Newman's equation [12]:

with

$$
\frac{\sigma_{\mathrm{op}}}{\sigma_{\max }}=\left\{\begin{array}{ccc}
A_{0}+A_{1} R+A_{2} R^{2}+A_{3} R^{3} & \text { for } & R \geq 0 \\
A_{0}+A_{1} R & \text { for } & R<0
\end{array}\right.
$$

$\mathrm{A}_{0}=0.535 \cos \frac{\pi \sigma_{\max }}{2 \sigma_{0}} ; \mathrm{A}_{1}=0.344 \frac{\sigma_{\max }}{\sigma_{0}} ; \mathrm{A}_{2}=1-\mathrm{A}_{0}-\mathrm{A}_{1}-\mathrm{A}_{3} ; \mathrm{A}_{3}=2 \mathrm{~A}_{0}+\mathrm{A}_{1}-1$

2. Calculation of strain at crack opening/closure for constant amplitude loading:

$$
\varepsilon_{\mathrm{op}, \mathrm{const}}=\varepsilon_{\mathrm{cl}, \mathrm{const}}=\varepsilon_{\mathrm{min}}+\frac{\sigma_{\mathrm{op}}-\sigma_{\mathrm{min}}}{E}+2\left(\frac{\sigma_{\mathrm{op}}-\sigma_{\mathrm{min}}}{2 K^{\prime}}\right)^{\frac{1}{n^{\prime}}}
$$

3. Calculation of the crack opening strain $\varepsilon_{\text {op }}$ depending on the crack opening strain of the previous load step $\varepsilon_{\text {op,previous: }}$

Special cases:

$$
\begin{aligned}
& \text { For } \varepsilon_{\max }>\varepsilon_{\text {max,previous }} \text { or } \varepsilon_{\text {min }}<\varepsilon_{\text {min,previous }} \rightarrow \varepsilon_{\text {op }}=\varepsilon_{\text {op,const }} \\
& \text { For } \varepsilon_{\max } \leq \varepsilon_{\text {op,previous }} \rightarrow \varepsilon_{\text {op }}=\varepsilon_{\text {op,previous }}
\end{aligned}
$$

Cases with $\varepsilon_{\text {op,const }} \geq \varepsilon_{\text {op,previous }} \rightarrow \varepsilon_{\text {op }}=\varepsilon_{\text {op,previous }}$

Cases with $\varepsilon_{\text {op,const }}<\varepsilon_{\text {op,previous }}$

For $\sigma_{\mathrm{a}} \geq 0.4 \sigma_{0} \rightarrow \varepsilon_{\text {op }}=\varepsilon_{\text {op,const }}$

For $\sigma_{\mathrm{a}}<0.4 \sigma_{0} \rightarrow \varepsilon_{\mathrm{op}}=\varepsilon_{\text {op,previous }}$

4. Calculation of the crack closure stress:

For $\varepsilon_{\mathrm{op}} \leq \varepsilon_{\mathrm{min}} \rightarrow \sigma_{\mathrm{cl}}=\sigma_{\text {min }}$

For $\varepsilon_{\mathrm{op}}>\varepsilon_{\mathrm{min}}$, the crack closure stress is iteratively determined from: 


$$
\varepsilon_{\mathrm{max}}-\varepsilon_{\mathrm{op}}=\frac{\sigma_{\mathrm{max}}-\sigma_{\mathrm{cl}}}{E}+2\left(\frac{\sigma_{\mathrm{max}}-\sigma_{\mathrm{cl}}}{2 K^{\prime}}\right)^{\frac{1}{n^{\prime}}}
$$

5. Calculation of damage parameter $\mathrm{P}_{\mathrm{J}}$ :

with

$$
P_{J}=\left[1.24 \frac{\Delta \sigma_{\mathrm{eff}}^{2}}{E}+\frac{1.02}{\sqrt{n^{\prime}}} \Delta \sigma_{\mathrm{eff}}\left(\Delta \varepsilon_{\mathrm{eff}}-\frac{\Delta \sigma_{\mathrm{eff}}}{E}\right)\right] .
$$

$$
\begin{aligned}
& \Delta \sigma_{\text {eff }}=\left(\sigma_{\max }-\sigma_{\mathrm{cl}}\right) \\
& \Delta \varepsilon_{\text {eff }}=\left(\varepsilon_{\max }-\varepsilon_{\mathrm{op}}\right)
\end{aligned}
$$

6. Calculation of the damage contribution $\mathrm{D}_{\text {actual }}$ and damage sum $\mathrm{D}_{\text {new }}$ :

$$
\begin{gathered}
D_{\text {actual }}=\frac{1}{N} \begin{cases}\frac{P_{\mathrm{J}}^{m}}{Q} & \text { for } \quad P_{\mathrm{J}} \geq P_{\mathrm{J}, \mathrm{e}} \\
0 & \text { for } \quad P_{\mathrm{J}}<P_{\mathrm{J}, \mathrm{e}}\end{cases} \\
D_{\text {new }}=D_{\text {previous }}+D_{\text {actual }}
\end{gathered}
$$

7. Calculation of endurance limit:

$$
P_{\mathrm{J}, \mathrm{e}}=P_{\mathrm{J}, \mathrm{e}, 0} \frac{a_{0}+l^{*}}{\left(\left(a_{\text {end }}^{1-m}-a_{0}^{1-m}\right) D_{\text {new }}+a_{0}^{1-m}\right)^{\frac{1}{1-m}}+l^{*}}
$$

8. Calculation of the crack opening strain for the following load cycle:

$$
\varepsilon_{\text {op,new }}=\varepsilon_{\text {op,const }}-\left(\varepsilon_{\text {op,const }}-\varepsilon_{\text {op,previous }}\right) e^{\left(-15 D_{\text {actual }}\right)}
$$

In step 3 the actual crack opening strain is either set to the previous crack opening strain (calculated by step 8) or to the crack opening strain for constant amplitude loading, depending on actual and previous loading. Generally, for two stage tests, the crack opening strain for high amplitude loadings is equal to the corresponding crack opening strain at constant amplitude loading, while it is mainly determined by previous load amplitudes for low amplitude loadings (and especially by the crack opening strain of the previous high amplitude loading). For a high amplitude loading followed by a various number of low amplitude loadings, the crack opening strain increases with an increasing number of low amplitude cycles, depending on the amount of damage for each load cycle (see step 8). Additionally, the load history is also taken into account by the reduction of the endurance limit (see step 7). With an increasing damage sum $\left(D_{\text {new }}\right)$, the initial endurance limit $P_{\mathrm{J}, \mathrm{e}, 0}$ is reduced according to the actual crack length.

\section{Results}

The data basis currently counts about 90 tests under variable amplitude loading for various specimen types and loadings. Under the assumption of Masing-Memory behaviour and a notch factor $\mathrm{K}_{\mathrm{f}}$ of 1.3 for notched and 1.95 for welded specimens (including notch transmission factor as well as statistical and fracture mechanical size effect) the comparison between experimental and calculated fatigue lives shows major differences between the individual damage calculation methods. The comparisons are shown in Fig. 3. The pure consideration of strain amplitudes led to fatigue lives which are in most cases higher than experimentally observed (see Fig 3 a, indicated by the mean value of ratio from calculated to experimental result). The highest deviations result for calculations with the damage parameter $\mathrm{P}_{\mathrm{SWT}}$, (see Fig $3 \mathrm{~b}$ ). It overestimates the mean stress effect and provides both too high as well as too low fatigue lives. In contrast, the damage parameter $\mathrm{P}_{\mathrm{J}}$ led to the most accurate calculation results. The accuracy can be improved by consideration of the following aspects: All damage calculations considered Masing-Memory behaviour, but the present austenitic steel 1.4550 shows a strong affinity to mean stress relaxation (see Fig. 4 left) and 


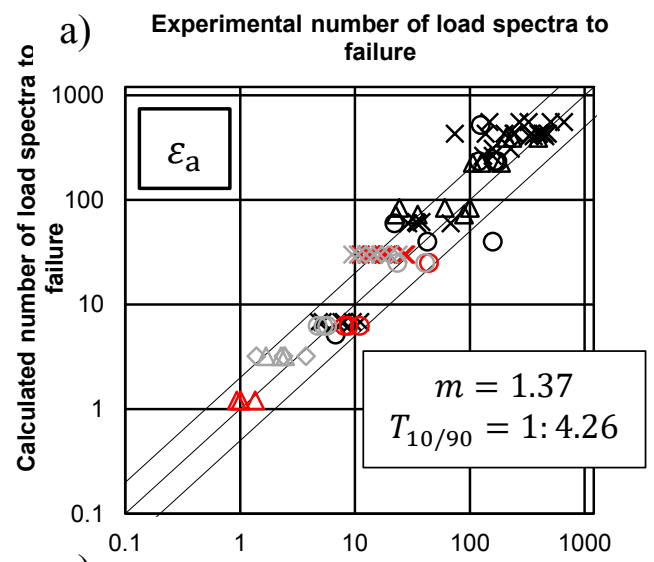

c)

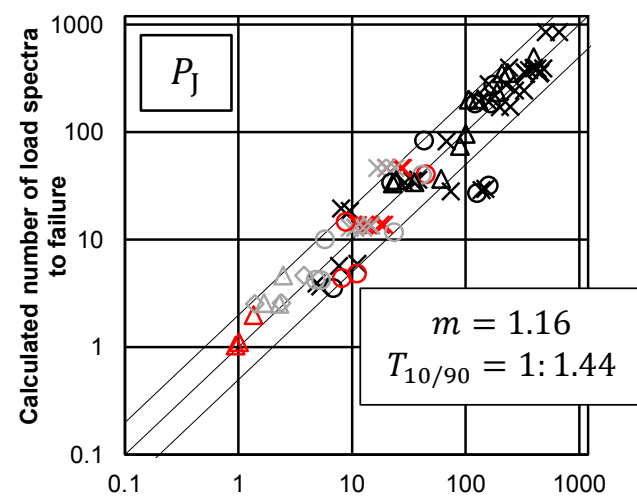
b) Experimental number of load spectra to

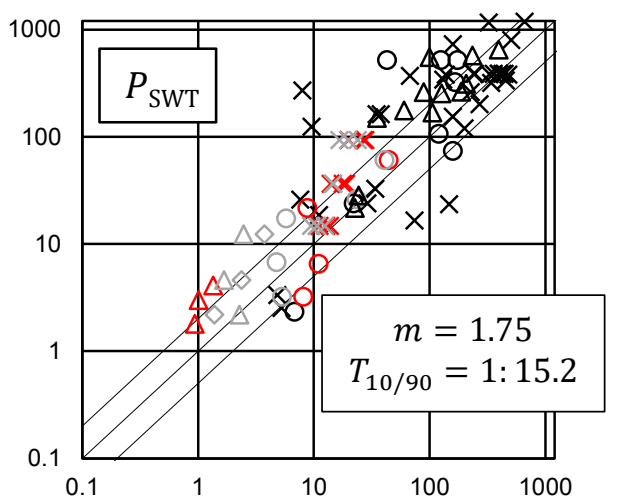

$\times$ HG-Specimen, Two Stage Loading

$\times$ HG-specimen, Eight Stage Loading

$\times$ HG-Specimen, Operational Loading

○ Notched Specimen, Two Stage Loading

○ Notched Specimen, Eight Stage Loading

- Notched Specimen, Operational Loading

$\Delta$ Welded Specimen, Two Stage Loading

$\Delta$ Welded Specimen, Eight Stage Loading

$\triangle$ Welded Specimen, Operational Loading

$\diamond$ Welded Specimen, Operational Loading

(TMF-Loading, $50-350^{\circ} \mathrm{C}$ )

Fig. 3. Comparison of experimental number of load spectra to failure and calculated number of load spectra to failure with pure strain amplitudes, damage parameter $\mathrm{P}_{\mathrm{SWT}}$ and damage parameter $\mathrm{P}_{\mathrm{J}}$ for Hourglass-, notched and welded specimens
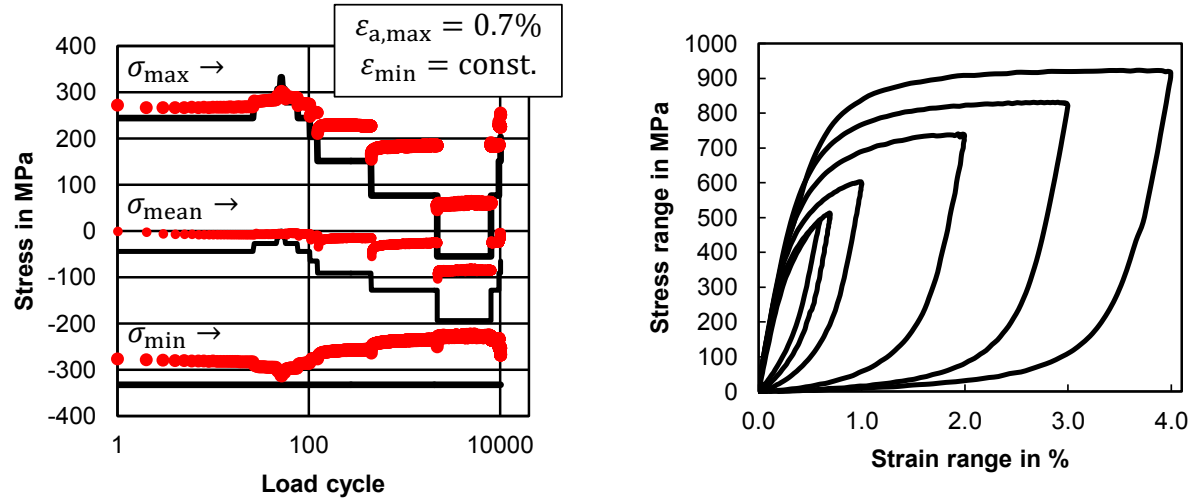

Masing-Memory $\bullet$ Experiment

Fig. 4. Experimentally observed maximum, minimum and mean stress for each cycle of an Eight-Block Test with smooth specimen in comparison with calculated maximum, minimum and mean stress by Masing-Memory (left); Stabilized hysteresis loops for strain controlled loading under constant amplitudes of $0.3 \%, 0.35 \%, 0.5 \%, 1.0 \%, 1.5 \%$ and $2.0 \%$ 
a Non-Masing behaviour (see Fig. 4 right). Mean stress relaxation led to shorter lives for a cycle in pressure and to longer lives for a cycle in tension. Considering these characteristics in damage calculation could improve the life prediction of damage parameter $\mathrm{P}_{\mathrm{J}}$ additionally.

\section{Conclusions and Outlook}

A broad test program to reveal different influences on variable amplitude loading of the austenitic steel 1.4550 and weld joints of this steel has been carried out with Hourglass-, notched and welded specimens. The measured load spectrum was ordered in blocks or as pseudo-random sequence as an operational load for the tests. Additionally, the placement of the amplitudes was varied $\left(\varepsilon_{\text {mean }}=\right.$ const., $\varepsilon_{\max }=$ const., $\varepsilon_{\min }=$ const. $)$. To assess the lifetime three different concepts have been used: Miner's rule with strain ranges, damage parameter $\mathrm{P}_{\mathrm{SWT}}$ and damage parameter $\mathrm{P}_{\mathrm{J}}$. Comparing the results of the various assessment methods, it can be concluded that $\mathrm{P}_{\mathrm{J}}$ gives the most accurate assessment of life. Nevertheless, the concept can be improved, especially the input data to the concept can be improved by consideration of the mean stress relaxation.

The IGF-project $18.842 \mathrm{~N}$ "Erweiterte Schädigungskonzepte für thermomechanische Beanspruchung unter variablen Amplituden und plastischer Deformation" of the research association "Schweißen und verwandte Verfahren e.V." of the DVS, Aachener Str. 172, 40223 Düsseldorf was funded by the AiF within the scope of the program for advancement of the industrial alliance of research (IGF) of the Federal Ministry for Economic Affairs and Energy on the basis of a decision by the German Bundestag. The financial support is greatly acknowledged.

\section{References}

1. T. Gilman, M. Gray, J. Rudolph, B. Heinz, Proc. of the ASME Pressure Vessels \& Piping Conference (Boston, 2015)

2. U. H. Clormann, T. Seeger, Stahlbau, 55, 65 (1986)

3. A. Palmgren, VDI-Z, 68, 339 (1924)

4. M. A. Miner, J. Appl. Mech., 12, A-159 (1945)

5. O. H. Basquin, Proc. ASTM 11, 625 (1910)

6. S. S. Manson, TN-2933 (NACA, 1953)

7. L. F. Coffin Jr., Transactions of the ASME, 76, 931 (1954)

8. K. N. Smith, P. Watson, T. H. Topper, J Materials, 5, 767 (1970)

9. P. C. Paris, Fatigue, An Interdisciplinary Approach, (Syracuse University Press, 1964)

10. N. E. Dowling, N. S. Iyyer, Low Cycle Fatigue and Elasto-Plastic Behaviour of Materials, 569, (Springer, 1987)

11. M. Vormwald, Ph.D Thesis (TH Darmstadt, 1989)

12. J. C. Newman, Design of Fatigue and Fracture Resistant Structures, 255, (ASTM, 1982)

13. M. Vormwald, Int J Fatigue, 82, 80 (2016)

14. D. Taylor, Compendium of Fatigue Thresholds and Growth Rates, Warley (West Midlands: Engineering Materials Advisory Services, 1985)

15. Y. Adonyi, Welding Journal, 85 (10), 42 (2006) 